\title{
Transcatheter balloon atrial septostomy in thoraco-omphalopagus conjoined twins
}

\author{
Gurumurthy Hiremath ${ }^{1}$, Lerraughn M. Morgan², Martina Richtsfeld ${ }^{3}$, Mojca Konia ${ }^{3}$, Daniel Saltzman ${ }^{4}$, \\ Matthew Ambrose ${ }^{1}$ \\ ${ }^{1}$ Department of Pediatrics, Division of Pediatric Cardiology, University of Minnesota, Masonic Children's Hospital, Minneapolis, MN, USA \\ 2Department of Pediatric Cardiology, Valley Children's Hospital, Fresno, CA, USA \\ ${ }^{3}$ Department of Pediatric Anesthesia, University of Minnesota, Masonic Childrens Hospital, Minneapolis, USA \\ ${ }^{4}$ Department of Pediatric Surgery, University of Minnesota, Masonic Children's Hospital, Minneapolis, USA
}

Adv Interv Cardiol 2020; 16, 2 (60): 209-212

DOI: https://doi.org/10.5114/aic.2020.96067

\section{Introduction}

Conjoined twinning is a rare congenital anomaly with a reported incidence of 1 in 50,000-200,000 births [1, 2]. Fusion at the thoracic level (thoracopagus) is the most common variety ( $40 \%$ of cases) followed by the abdomen (omphalopagus, 32\%) [1]. Cardiac anomalies are common in these patients and range from shared pericardium to fused atria and ventricles [1]. Congenital heart disease (CHD) is common in addition to cardiac fusion in these children [3]. Perinatal outcomes are determined by the severity of cardiac involvement. Overall survival for conjoined twins is dismal at 25\% [4]. Survival after successful separation for a conjoined twin with fused hearts is even more rare, with reported survival of $10 \%$ if fused at the atrial level and $0 \%$ if fused at the ventricular level $[3,5]$.

Cardiac catheterization has been used in diagnosis of CHD in these patients [3]. We report a case of thoraco-omphalopagus twins with atrial fusion, where the index twin had CHD and required transcatheter balloon atrial septostomy (BAS) at 3 months of age prior to surgical separation. Balloon atrial septostomy played a key role in medical management and successful cardiac surgery.

\section{Case report}

There was prenatal diagnosis of thoraco-omphalopagus conjoined twinning of two female fetuses with union from just cranial to the manubria, to just caudal to a common umbilicus, with a shared liver but otherwise separate intraabdominal and intrathoracic contents. Fetal echocardiography and post-natal transthoracic echo- cardiography identified separate cardiac masses with a shared pericardial sac. In this case, the index twin (twin B) had levocardia with a levoposed cardiac apex, tricuspid atresia with dextro-transposition of the great vessels and a large bulboventricular foramen, and secundum atrial septal defect. The co-twin (twin A) had dextroversion of the heart, but otherwise normal cardiac structure. In addition, there was a small vermiform vascular communication between the right atrial appendage of the index twin and the dextroverted/anterior left atrium of the co-twin. Flow across this tiny connection could only be demonstrated consistently well in the fetal echocardiograms. The twins were born by Caesarean section at $33+6 / 7$ weeks gestational age.

The hemodynamics in the conjoined pair were complex. Using a combination of transthoracic echocardiography and CT angiography, arterial communications between the twins in the hepatic artery and internal thoracic and mammary arteries were demonstrated, with unidirectional flow from the index twin to the co-twin. Extensive intrahepatic venous connections were identified, and conversely were unidirectional from co-twin to index twin. This resulted in a unique shared physiology where the co-twin received an increased share of the total preload between the two hearts, and was generating an increased share of the total cardiac output.

The atrial septal defect in the index twin became progressively more restrictive in the first 3 months of life. This resulted in less and less contribution to the combined cardiac output from the index twin through decreased preload, and consequently additional shunting of systemic venous return to the co-twin through the

\section{Corresponding author:}

Gurumurthy Hiremath, Department of Pediatrics, Division of Pediatric Cardiology, University of Minnesota, Masonic Children's Hospital, Minneapolis, MN, USA, phone: +16126262467 , e-mail: hiremath@umn.edu

Received: 15.04.2020, accepted: 25.04.2020. 

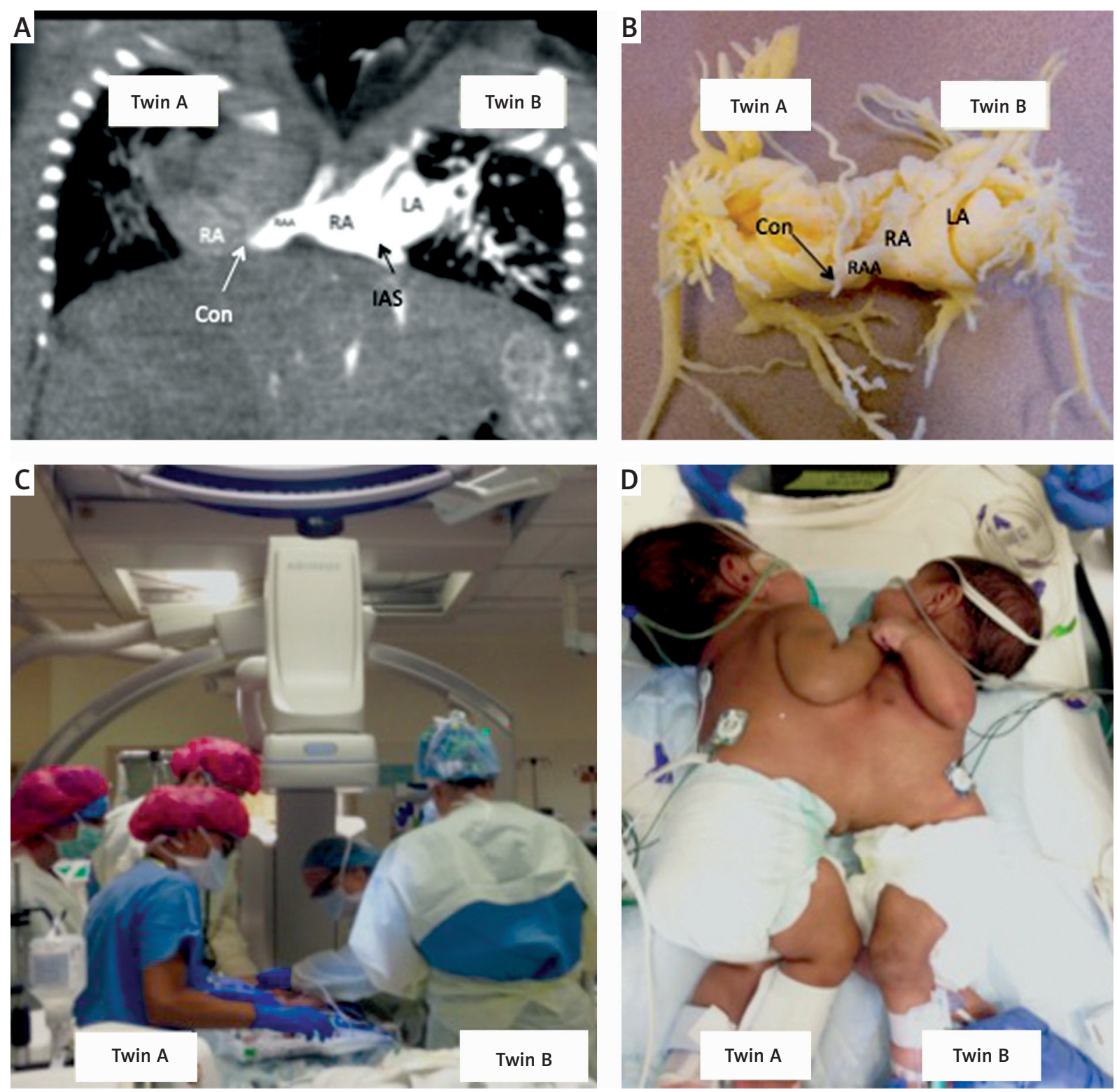

Figure 1. CT Angiogram sagittal view (A) and 3D printed model (B) demonstrating the restrictive inter atrial septum (IAS) and the relationship between twin A and twin B. The tiny atrial fistula (Con) had barely any flow in postnatal life and arose from the enlarged right atrial appendage (RAA): C, D - the set up in the catheterization laboratory. Twin A team wearing red hats and Twin B wearing blue hats. Twin A is to the left, Twin B to the right (B)

$L A$ - left atrium, $R A$ - right atrium.

intrahepatic venous connections. There was no demonstrable shunting through the tiny vermiform atrial fistula between the two twins at this point. As a result, the cotwin became increasingly more responsible for systemic blood flow in the index twin through shunting in the hepatic and thoracic/internal mammary arteries. The index twin developed significant oliguria and relative hypotension, and the co-twin developed polyuria and systemic hypertension presumably owing to the additional cardiac output. Twin A experienced hypertension at approximately 4 weeks of life requiring the initiation of nicardipine. To maintain adequate blood pressure in the setting of shared vascular supply, twin B was started on dopamine.

There was tremendous planning in preparation for the cardiac catheterization. Challenges included: 1) accommodating two anesthesia teams with two sets of personnel, equipment and monitors in the cardiac catheterization suite along with catheterization equipment and echocardiography machine; 2) planning for proper positioning of the twins so that there was easy access to the groin for access; 3) medication dosing and airway management since cross circulation between both twins meant all medications reached both twins simultaneous- 
ly. A practice run was done two days prior to the procedure. A three-dimensional model of the heart, which was created from the CT scans of the twins (Figure 1), helped in understanding the anatomy better and planning proper positioning in the catheterization laboratory. Hemodynamics and blood flow between the twins are depicted in Figure 2.

The twins were taken to the cardiac catheterization laboratory with two anesthesia teams and two anesthesia workspaces. Each team was color-coded with the Twin A team wearing red hats and the Twin B team wearing blue hats (Figure 1). Induction of anesthesia was simultaneous with sequential intubation of the twins. Both twins were intubated and mechanically ventilated. After preparation was completed by the anesthesia team, the twins were positioned on the catheterization table with the index twin on the right side. The co-twin was on the left side and positioned at a higher level using wedges for easier access to the index twin's groin. Cardiac catheterization was performed under echocardiographic and fluoroscopic guidance. Access was obtained in the index twin through the right femoral vein. The atrial septum was crossed using an angled glide catheter, and dynamic balloon atrial septostomy was performed using 9.5 and $10.5 \mathrm{~mm}$ B. Braun atrial septostomy balloons, followed by static balloon dilation with a $12 \mathrm{~mm}$ and $20 \mathrm{~mm}$ Tyshak II balloon. In the author's experience, static balloon dilation after initial conventional balloon septostomy helps further enlarge the atrial communication, especially beyond the neonatal period, where the septum is usually thick. This resulted in a large atrial level communication. During cardiac catheterization, right atrial saturation improved from $53 \%$ to $73 \%$ and left atrial saturation improved from $94 \%$ to $99 \%$, indicating improved admixture at the atrial level. Left atrial and right atrial filling pressures were borderline high and equal prior to BAS without significant post-BAS change, which was likely secondary to continued decompression of the right atrium through the lower pressure liver vascular supply (shared between infants). There was an immediate change in the hemodynamics with echocardiographic evidence of increased antegrade flow from the index twin's aorta. There were no complications.

In the subsequent week following BAS, there was a significant improvement in urine output from the index twin (0.3-1 ml/kg/h before BAS to $0.9-1.9 \mathrm{ml} / \mathrm{kg} / \mathrm{h}$ postBAS). There was no appreciable change in the degree of systemic hypertension or polyuria in the co-twin. The twins underwent successful surgical separation a week later along with ligation and division of the atrial connection. The index twin underwent pulmonary artery banding to protect the pulmonary bed from over-circulation immediately after separation. The co-twin was successfully discharged from hospital on day-of-life 284 . The index twin ultimately underwent tracheostomy on dayof-life 245 , was also successfully discharged from hospi-

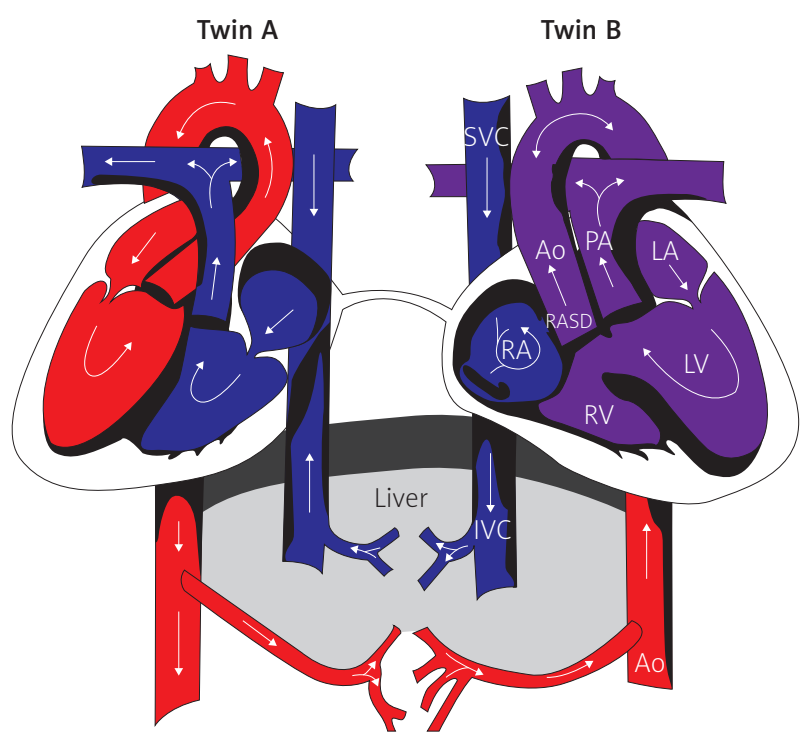

Figure 2. Hemodynamics and blood flow changes with restriction of flow across the atrial septum in Twin 2 (Index twin). Arrows indicate direction of blood flow

$S V C$ - superior vena cava, IVC - inferior vena cava, $R A$ - right atrium, RASD - restrictive atrial septal defect, Ao - aorta, PA - pulmonary artery, LA - left atrium, LV - left ventricle.

tal on day-of-life 417, subsequently underwent a Glenn procedure as an outpatient and at the time of writing is doing well at home.

Our case illustrates the importance of thorough pre-surgical work up and planning. A good understanding and analysis of vascular connections, hemodynamics and intricate cross-circulation patterns provided valuable information about hemodynamic behavior before after the catheterization procedure. Careful multidisciplinary planning was critical in the successful heart catheterization procedure.

\section{Conclusions}

This detailed report described successful percutaneous, transcatheter BAS in a conjoined twin prior to separation, resulting in improved hemodynamics, survival of the index twin and eventually paving the way for their successful surgical separation. We hope multidisciplinary teams can utilize our case to perform procedures in the cardiac catheterization laboratory for patients with similar clinical scenarios.

\section{Conflict of interest}

The authors declare no conflict of interest.

\section{References}

1. Ambar SS, Halkati PC, Patted SV, Yavagal S. Twin heart with a fused atria and separate ventricles in conjoined twins. Ann Pediatr Cardiol 2010; 3: 196-8. 
2. Edmonds LD, Layde PM. Conjoined twins in the United States, 1970-1977. Teratology 1982; 25: 301-8.

3. McMahon CJ, Mullins CE, Vick GW $3^{\text {rd }}$, et al. Cardiac catheterization in diagnosis and management of congenital heart disease in thoracopagus conjoined twins. Catheter Cardiovasc Interv 2000; 51: 159-67.

4. Stone JL, Goodrich JT. The craniopagus malformation: classification and implications for surgical separation. Brain 2006; 129: 1084-95.

5. Chiu CT, Hou SH, Lai HS, et al. Separation of thoracopagus conjoined twins. A case report. J Cardiovasc Surg 1994; 35: 459-62. 